\title{
Number of Compatible Pair of Actions for Finite Cyclic Groups of 3-Power Order
}

\author{
Mohammed Khalid Shahoodh ${ }^{\mathrm{a}}$, Mohd Sham Mohamad ${ }^{\mathrm{b}}$, Yuhani Yusof ${ }^{\mathrm{c}}$ and Sahimel Azwal Sulaiman ${ }^{\mathrm{d}}$ \\ Applied \& Industrial Mathematics (AIMs) Research Cluster, Faculty of Industrial Sciences \& Technology \\ Universiti Malaysia Pahang, Kuantan, Malaysia. \\ ${ }^{\mathrm{a}}$ moha861122@yahoo.com, ${ }^{\mathrm{b}}$ mohdsham@ump.edu.my, ${ }^{\mathrm{c}}$ yuhani@ump.edu.my, ${ }^{\mathrm{d}}$ titus1704@yahoo.com
}

\begin{abstract}
Compatible actions are important in determining the non-abelian tensor product. Different compatible pair of actions gives a different tensor product even for the same group. The purpose of this paper is to determine the exact number of compatible pair of actions for the finite cyclic groups of 3-power order. By using some properties of number theory, the number of the compatible pair of actions for finite cyclic groups of 3-power order with a specific order of actions is determined and given as a main result in this paper.
\end{abstract}

Keywords - Non-abelian Tensor Product, Auto-morphism Group, Cyclic Groups, Number Theory.

\section{INTRODUCTION}

The non-abelian tensor product of groups denoted by $G Q H$ which is defined for the pair of groups $G$ and $H$ providing the groups act on each other. The concept of the tensor product started in connection with a generalized Van Kampen theorem and the structure of this concept has its origins in the algebraic K-theory and also in the homotopy theory. The non-abelian tensor product of groups with compatible actions introduced by Brown and Loday [1] which extending the ideas of Whitehead [2]. Let $G$ and $H$ be groups, then the non-abelian tensor product of groups is the group generated by the symbols $g \otimes h$ with the relations:

$$
\begin{gathered}
g g^{t} \otimes h=\left(g_{g} \otimes g_{h}\right)(g \otimes h), \\
g \otimes h h^{t}=(g \otimes h)\left(h_{g} \otimes h_{h}\right)
\end{gathered}
$$

and satisfy the compatibility conditions:

$$
\begin{aligned}
& { }^{g} h g^{l}=g\left(g^{h}\left(g^{-1} g^{t}\right)\right) \text { and } \\
& \left.{ }^{h} g\right) h^{t}={ }^{h}\left(g^{g}\left(h^{-1} h^{t}\right)\right)
\end{aligned}
$$

for each $g_{n} g^{l} \in G$ and $h_{z} h^{l} \in H$.

Brown et. al [3] have studied group theoretical properties and the explicit computation of the non-abelian tensor product and provided eight open problems regarding the non-abelian tensor product and the nonabelian tensor square. In this research, we are interested with the concept of the non-abelian tensor product where the actions act compatibly on each other.

Ellis and McDermott [4] have checked the compatible actions for the two different groups $D_{4}$ and $Q_{8}$ when calculating the non-abelian tensor products. They found that only 292 compatible pair of actions between $D_{4}$ and
$Q_{\mathrm{g}}$. Visscher [5] used the cyclic groups of $p$-power order and provided necessary and sufficient number theoretical conditions for a pair of cyclic groups to act compatibly. In addition, Visscher [5] gave complete compatible conditions when one of the actions is trivial or both actions are trivial. Mohamad [6] represents the new necessary and sufficient number theoretical conditions and the characterization that a pair of finite cyclic groups of the $p$-power order acts compatibly on each other with the conditions depend on the order of the action. A paper by Mohamad et. al [7] gave the compatibility and the nonabelian tensor product of cyclic groups of order $p^{2}$ with the actions of order $p$. Meanwhile, Sulaiman et. al [8] investigated the compatible pairs of nontrivial actions of order two and four for the finite cyclic groups of 2-power order. In [9], Shahoodh et. al determined the compatible pair of nontrivial actions for cyclic groups of 3-power order. However, they gave some necessary and sufficient number theoretical conditions for a pair of finite cyclic groups of 3-power order with nontrivial actions to act compatibly on each other with the actions that have order three and nine satisfy the compatibility conditions. Besides that, Sulaiman et. al [10] have studied the compatible pair of nontrivial actions for two same finite cyclic 2-groups of 2-power order and provided the exact number of the compatible pair of nontrivial actions for such type groups with the actions that have a same order. In [11], Sulaiman et. al also determined the exact number of the compatible pair of actions for the finite cyclic 2groups when one of the actions has order greater than two. In this paper, the aim is to provide the exact number of the compatible pair of actions focusing only on the finite cyclic groups of 3-power order. Groups, Algorithm and Programming (GAP) software [12] is used to compute the compatible actions for the finite cyclic groups of 3-power order. 
In section 2, some definitions and related results on the auto-morphism of the finite cyclic groups of $p$-power order and the compatible of actions are given. Then, the number of the compatible pair of actions for the finite cyclic groups of 3-power order is given in section 3 . Lastly, the conclusions of this paper are given in section 5.

\section{THE PREPARATORY RESULTS}

In this section, some definitions and related results on the auto-morphism of finite cyclic groups of $p$-power order, number theory and the compatible of actions that will be used to prove the new results are given. We started with definitions of the action and compatible actions that are given as follows:

Definition 2.1 [5]

Let $G$ and $H$ be cyclic groups. An action of $G$ on $H$ is a mapping, $\Phi_{1} G \rightarrow \operatorname{Aut}(H)$ such that:$$
\Phi\left(g g^{l}\right)(h)=\Phi(g)\left(\Phi\left(g^{t}\right)(h)\right)
$$$$
\text { for all } g, g^{t} \in G \text { and } h \in H \text {. }
$$

Definition 2.2 [5]

Let $G$ and $H$ be groups which act on each other and each of which acts on itself by conjugation. Then the actions are compatible if:

$$
\begin{gathered}
\left.\left.{ }^{g} h\right) g^{t}=g\left({ }^{h}\left(g^{-4} g^{t}\right)\right)\right) \text { and } \\
\left.\left(^{b} g\right) h^{t}={ }^{h}\left(g^{g}\left(h^{-1} h^{b}\right)\right)\right]
\end{gathered}
$$

for all $g, g^{l} \in G$ and $h_{r} h^{l} \in H_{\text {. }}$.

In order to find the number of the actions, Euler's $\varphi$ function is needed and stated in the following definition.

\section{Definition 2.3 [14] Euler's $\varphi$-function}

For, $\geq 1$, the Euler's Phi-function, denoted by $\varphi(m)$, is the number of the positive integers not exceeding $m$ that are relatively prime with $m$.

Dummit and Foote in [11] showed that the automorphism of the finite cyclic group of $p$-power order is an isomorphic with a direct product of two finite cyclic groups as stated in following theorem.

Theorem 2.1 [13] Let $p$ be an odd prime and $\alpha \in \mathbb{Z}^{+}$. If $G$ is a cyclic group of order $p^{\alpha}$, then $\operatorname{Aut}\left(C_{p^{\alpha}}\right) \cong C_{p-1} \times C_{p^{\alpha-2}} \cong C_{(p-1) p^{\alpha-2} \text { and }}$ $\left|A u t\left(C_{p^{\alpha}}\right)\right|=\varphi\left(p^{\alpha}\right)=(p-1) p^{\alpha-1}$.
Visscher [5] shows in the following corollary that if $G$ is abelian group, then every pair of actions are compatible when one of the actions is trivial.

Corollary 2.1 [5] Let $G$ and $H$ be groups. Furthermore, let $G$ act trivially on $H$. If $G$ is abelian, then for any action of $H$ on $G$ the mutual actions are compatible.

Next, Mohamad [6] provided a new necessary and sufficient number theoretical conditions for two finite cyclic groups of $p$-power order where $p$ is an odd prime to act compatible on each other with the conditions depend on the order of the action. The result is given in the following theorem.

Theorem 2.2 [6] Let $G=\langle g\rangle \cong C_{p} \alpha$ and $H=\langle h\rangle \cong C_{p^{p}} \quad$ where $\quad \alpha, \beta \geq 2$. Furthermore, let $\sigma \in \operatorname{Aut}(G) \quad$ with $\quad|\sigma|=p^{k} \quad$ where $k=1,2, \ldots, \alpha-1 \quad$ and $\sigma^{i} \in \operatorname{Aut}(H) \quad$ with $\left|\sigma^{t}\right|=p^{k^{t}}$ where $k^{t}=1,2, \ldots, \beta-1$. Then $\left(\sigma_{v} \sigma^{t}\right)$ is a compatible pair if and only if $k+k^{k} \approx \min \left\{\alpha_{r} \beta\right\}$.

In the next section, all the results for the number of the compatible pair of actions for the finite cyclic groups of 3power order are given.

\section{RESULTS AND DISCUSSION}

In this section, the number of the compatible pair of actions for a pair of the finite cyclic groups of 3-power order is determined by using some properties of number theory for such type groups. Since, the cyclic group is considered, then an action of a group $G$ on a group $H$ is the homomorphism from $G$ to $\operatorname{Aut}(H)$ and an action of a group $H$ on a group $G$ is the homomorphism from $H$ to $\operatorname{Aut}(G)$. Hence, the number of the auto-morphisms needs to be find first. Thus, the number of the auto-morphisms for the finite cyclic groups of 3-power order with the respective order is given in the following proposition.

Proposition 3.1 Let $G=\langle x\rangle \cong C_{g^{\alpha}}$ be finite cyclic group of 3-power order with $\alpha \in \mathbb{N}$. Then, there exist $2 \cdot 3^{k-1}$ auto-morphisms of order $3^{k}$ where $k=1,2, \ldots, \alpha-1$.

Proof: Let $G=\langle x\rangle \cong C_{g^{\alpha}}$ be finite cyclic group of 3-power order with $\alpha \in \mathbb{N}$. Without loss of generality, suppose that $H$ be a finite cyclic $p$-subgroup of $G$ such that $|H|=3^{k}$ where $k=1,2_{, \ldots,} \alpha-1$. Thus, each 
element that relatively prime with $3^{k}$ has an order $3^{k}$. Since $H$ be a cyclic $p$-subgroup, then by using Phifunction we have $\varphi\left(3^{k}\right)=(3-1) 3^{k-1}=2 \cdot 3^{k-1}$ which is the number of the auto-morphism that have order $3^{k}$.

Now, the number of the auto-morphisms of the finite cyclic groups of 3-power order that having order 3 is given in the following corollary.

Corollary $3.1 G=\langle x\rangle \cong C_{g} \approx$ Let be finite cyclic group of 3-power order with $\alpha \in \mathbb{N}$. Then, there exist two auto-morphisms that have order three.

Proof: Let $G=\langle x\rangle \cong C_{g} \approx$ be finite cyclic group of 3-power order with $\alpha \in \mathbb{N}$. By Proposition 3.1 for any cyclic group of 3 -power order there are $2 \cdot 3^{k-1}$ automorphism that have order $3^{k}$ where $k=1,2, \ldots-1$. Thus, for $k=1$, we have $2 \cdot 3^{1-1}=2$ which is the number of the automorphisms of order 3 .

The following corollary gives the number of the automorphisms of the finite cyclic groups of 3-power order that having the highest 3 -power order which is $3^{\propto-1}$.

Corollary 3.2 Let $G=\langle x\rangle \cong C_{\mathrm{g}} \alpha$ be finite cyclic group of 3-power order with $\alpha \in \square$. Then, there exist $2 \cdot 3^{\alpha-2}$ auto-morphisms that have order $3^{\alpha-1}$.

Proof: Let $G=\langle x\rangle \cong C_{9} \approx$ be finite cyclic group of 3-power order with $\alpha \in \mathbb{N}$. By Proposition 3.1 for any cyclic group of 3 -power order there are $2 \cdot 3^{k-1}$ automorphism that have order $3^{k}$ where $k=1,2, \ldots-1$. Thus, for $k=\alpha-1$, we have $(3-1) 3^{\alpha-1-1}=2 \cdot 3^{\alpha-2}$ which is the number of the auto-morphisms of order $3^{\alpha-1}$.

Now, the number of the compatible pair of actions for the finite cyclic groups of 3-power order has been determined. First, suppose that one of the actions has an order one, then the number of the compatible pair of actions is given in the following proposition.

Proposition 3.2 Let $G \cong C_{g}$ and $H \cong C_{g}$ be finite cyclic groups of 3-power order. Furthermore, let $\rho \in \operatorname{Aut}(G)$ with $|\rho|=1$ and $\rho^{i} \in \operatorname{Aut}(H)$, where $\alpha, \beta \geq 1$. Then the number of the compatible pair of actions is $2 \cdot 3^{\beta-1}$.

Proof: Let $G \cong C_{9} a$ and $H \cong C_{0} F$. Furthermore, let $\rho \in \operatorname{Aut}(G) \quad$ with $|\rho|=1 \quad$ and $\beta^{i} \in \operatorname{Aut}(H)$, where $\alpha_{r} \beta \geq 1$. By Corollary 2.1, when $G$ act trivially on $H$, then any action of $H$ on $G$ the mutual actions are compatible. Thus, by Theorem 2.1,
$|A u t(H)|=(3-1) 3^{\beta-1}=2 \cdot 3^{\beta-1}$ be the number of the compatible pair of actions.

Next, the number of the compatible pair of the actions for such type groups where one of the actions has an order three is determined. This result is given in the following proposition.

Proposition 3.3 Let $G \cong C_{9} \alpha$ and $H \cong C_{g}$ be finite cyclic groups of 3-power order. Furthermore, let $\rho \in \operatorname{Aut}(G)$ with $|\rho|=3$ and $\rho^{i} \in \operatorname{Aut}(H)$, where $\alpha, \beta \geq 3$. Then the number of the compatible pair of actions is $6+2\left(3^{r-1}-3\right)$ where $r^{r}=\min \{\alpha, \beta\}$.

Proof: Let $G \cong C_{3^{\alpha}}$ and $H \cong C_{g}$. Furthermore, let $\rho \in \operatorname{Aut}(G)$ with $|\rho|=3$ and $\rho^{t} \in \operatorname{Aut}(H)$, where $\alpha, \beta \geq 3$. First, consider $\left|\beta^{t}\right|=1$, by Corollary 2.1 the actions are compatible with every finite cyclic group of 3power order that has $(3-1)$ auto-morphism of order 3 . By Corollary 3.1 There are two compatible pair of actions under this case. Next, consider, $\left|\rho^{t}\right|=3$, by Theorem 2.2, the actions are compatible. By Corollary 3.1 there are two auto-morphisms of both groups $G$ and $H$. Thus there are $2^{2}$ compatible pair of actions. Finally, let $\left|\rho^{t}\right|=3^{k}$ where $k=2_{v \ldots,}, \alpha-1$. By Theorem $2.2\left(\rho_{v} \rho^{l}\right)$ are compatible. Thus, we have $(3-1)\left(3^{r-1}-3\right)$ compatible pair of actions where $r=\min \{\alpha, \beta\}$.

Finally, in total there are $2+4+2\left(3^{r-1}-3\right)=6+2\left(3^{r-1}-3\right)$ compatible pair of actions where $r=\min \{\alpha, \beta\}$.

The following proposition gives the number of the compatible pair of actions for the finite cyclic groups of 3power order where one of the actions has an order $3^{\alpha-1}$.

Proposition 3.4 Let $G \cong C_{3^{\alpha}}$ and $H \cong C_{3^{\beta}}$ be finite cyclic groups of 3-power order. Furthermore, let $\rho \in \operatorname{Aut}(G)$ with $|\rho|=3^{\alpha-1}$ and $\rho^{t} \in \operatorname{Aut}(H)$, where $\alpha, \beta \geq 3$. Then the number of the compatible pair of actions is $6 \cdot 3^{\kappa-1}$.

Proof: Let $G \cong C_{g^{\alpha}}$ and $H \cong C_{3^{*}}$ be finite cyclic groups of 3-power order. Furthermore, let $\rho \in \operatorname{Aut}(G)$ with $|\rho|=3^{\alpha-1}$ and $\rho^{\gamma} \in \operatorname{Aut}(H)$, where $\alpha_{v} \beta \geq 3$. By Theorem 2.2 the actions are compatible when $\left|\rho^{t}\right|=1$ and $\left|\rho^{t}\right|=3$. First, consider $\left|\rho^{t}\right|=1$, by Corollary 2.1 when one of the actions is trivial the actions are compatible. Thus, by Corollary 3.2 there are $2 \cdot 3^{\alpha-2}$ 
compatible pair of actions under this case. Finally, let $\left|\rho^{t}\right|=3$, by Theorem 2.2 , we have $(2)^{2} \cdot 3^{\alpha-2}$ compatible pair of actions in this case. Therefore, when one of the actions has an order $3^{\alpha-1}$, there are $2 \cdot 3^{\alpha-2}+(2)^{2} \cdot 3^{\alpha-2}=6 \cdot 3^{\alpha-2}$ compatible pair of actions.

Now, the results have been discovered on the possible cases to find the number of the compatible pair of actions between two finite cyclic groups of 3-power order which gives the maximum non-abelian tensor product for the cyclic groups of 3-power order. The order of the actions which are order one, order three and the highest 3-power order are considered the main point in the results to determine the number of the compatible pair of actions. Besides that, the number of the auto-morphisms that have a 3-power order has been also determined and used to find the exact number of compatible pair of actions for such type groups.

\section{CONCLUSION}

As a conclusion, the exact number of the compatible pair of actions for the finite cyclic groups of 3-power order has been given according to the order of the actions that satisfying the compatibility conditions. By using some properties of number theory with specific order of actions for such type groups, the number of the compatible pair of actions has been determined.

\section{REFERENCES}

[1] Brown, R. and Loday, J. L. 1984. Excision Homotopique En Basse Dimension. C.R. Acad. Sci. Ser. I Math. Paris. 298: 353356.

[2] Whitehead, J.H.C. 1950. A Certain Exact Sequence. Annals of Mathematics. 51: 51-110.

[3] Brown, R., Johnson, D. L. and Robertson, E. F. 1987. Some computations of non-abelian tensor products of Groups. J. of Algebra. 111: 177-202.

[4] Ellis, G. and McDermott, A. 1998. Tensor products of prime power Groups. J. Pure Applied Algebra. 132: 119-128.

[5] Visscher, M. 1998. On the non-abelian tensor products of groups. PhD Dissertation, State University of New York, Binghamton, NY.

[6] Mohamad, M. S. 2012. Compatibility conditions and non-abelian tensor products of finite cyclic groups of $p$-power order. $\mathrm{PhD}$ Dissertation, Universiti Teknologi Malaysia.

[7] Mohamad, M. S., Sarmin, N. H., Ali, N. M. M., and Kappe, L. C. 2012. The computation of the non-abelian tensor product of cyclic groups of order $p^{2}$.Journal Teknologi (Sciences \& Engineering) Penerbit UTM Press, Universiti Technologi Malaysia. 57: 35-44.

[8] Sulaiman, S. A., Mohamad, M. S., Yusof, Y., Sarmin N. H., Ali, N. M. M., Ken, T. L., \& Ahmad, T. 2015. Compatible pair of nontrivial actions for some cyclic groups of 2-power order. In The 2nd Ism International Statistical Conference 2014 (ISM-II): Empowering The Applications of Statistical And Mathematical Sciences. 1643:700-705. AIP Publishing.

[9] Shahoodh, K. M., Mohamad, M. S., Yusof., Y \& Sulaiman, S., A. 2016. Compatible Pair of Nontrivial Actions for Cyclic Groups of 3-Power Order. The National Conference for Postgraduate Research. Universiti Malaysia Pahang. p 108, pp 792-797.

[10] Sulaiman, S. A., Mohamad, M. S., Yusof, Y \& Shahoodh, K. M., 2016. Compatible Pair of Nontrivial Action For Finite Cyclic 2Groups. The National Conference for Postgraduate Research 2016, Universiti Malaysia Pahang. p005, pp39-42.

[11] Sulaiman, S. A., Mohamad, M. S., Yusof, Y., Shahoodh, K. M., 2016. Number of Compatible Pair For Nontrivial Actions of Finite Cyclic 2-Group. Proceedings of 4thInternational Science Postgraduate Conference 2016 (ISPC2016) (C) Faculty of Science, Universiti Teknologi Malaysia.

[12] http://www.gap-system.org. The GAP Group, GAP-Groups, Algorithm, and programming, Version 4.7, 2015.

[13] Dummit D. S., and Foote R. M., Abstract Algebra, USA: John Wiley and Sons, 2004.

[14] Burton, D. Elementary Number Theory. Sixth Edition. USA: McGraw Hill 2005. 